\title{
Effect of grafting on properties of oil palm empty fruit bunch fiber reinforced polycaprolactone biocomposites
}

\begin{abstract}
Biocomposites of polycaprolactones (PCL) and oil palm empty fruit bunch fiber (OPEFB) were prepared and characterized. In order to improve the mechanical properties the biocomposites, the OPEFB was modified by grafting with methyl methacrylate (MMA). The modification of fiber was carried out at $70^{\circ} \mathrm{C}$ under nitrogen atmosphere by using hydrogen peroxide as an initiator and $\mathrm{Fe} 2+$ as catalyst. Fourier transform infrared spectroscopy analysis showed that, after modification, the $\mathrm{OH}$ stretching in the sample was decreased, while the $\mathrm{C}=\mathrm{O}$ absorption was increased. The grafted and the ungrafted OPEFB were used to prepare PCL/OPEFB-g-PMMA and PCL/OPEFB biocomposites. The tensile and flexural strength of PCL/OPEFB-g-PMMA composite were improved, indicating good interaction between OPEFB-g-PMMA and PCL. An improvement of impact strength also can be seen in the PCL/OPEFB-g-PMMA composite. The micrographs of the tensile specimens of the fractured surface examined by the scanning electron microscopy demonstrated better adhesion between the grafted OPEFB and PCL.
\end{abstract}

Keyword: PCL; OPEFB; Biocomposites; Mechanical properties; Grafting, Monomer 